\begin{tabular}{|c|l|}
\hline Title & $\begin{array}{l}\text { Overexpression of TNF-a converting enzyme promotes adipose tissue inflammation and fibrosis induced by high fat } \\
\text { diet }\end{array}$ \\
\hline Author(s) & $\begin{array}{l}\text { Matsui, Y Yki; Tomaru, Utano; Miyoshi, A rina; Ito, Tomoki; Fukaya, Shinji; Miyoshi, Hideaki; A tsumi, Tatsuya; Ishizu, } \\
\text { A kihiro }\end{array}$ \\
\hline Citation & $\begin{array}{l}\text { Experimental and Molecular Pathology, 97(3), 354.358 } \\
\text { https://doi.org/10.1016/.yexmp.2014.09.017 }\end{array}$ \\
\hline Issue Date & 201412 \\
\hline Doc URL & http://hdl.handle.net/2115/57002 \\
\hline Type & article (author version) \\
\hline File Information & Accepted Manuscript_Matsui et al_Exp Mol Pathol-2.pdf \\
\hline
\end{tabular}

Instructions for use 


\section{Overexpression of TNF- $\alpha$ converting enzyme promotes adipose tissue inflammation and fibrosis induced by high fat diet}

Short title: TACE and adipose tissue inflammation

Yuki Matsui ${ }^{1}$, Utano Tomaru ${ }^{2}$, Arina Miyoshi ${ }^{3}$, Tomoki Ito $^{2}$, Shinji Fukaya ${ }^{3}$,

Hideaki Miyoshi ${ }^{3}$, Tatsuya Atsumi ${ }^{3} \&$ Akihiro Ishizu $^{4}$

${ }^{1}$ Graduate School of Health Sciences, Hokkaido University, Sapporo, Japan

${ }^{2}$ Department of Pathology, Hokkaido University Graduate School of Medicine, Sapporo, Japan

${ }^{3}$ Department of Internal Medicine II, Hokkaido University Graduate School of Medicine, Sapporo, Japan

${ }^{4}$ Faculty of Health Sciences, Hokkaido University, Sapporo, Japan

Address correspondence and reprint requests to Akihiro Ishizu, $\mathrm{MD}, \mathrm{PhD}$

Faculty of Health Sciences, Hokkaido University

Kita-12, Nishi-5, Kita-ku, Sapporo 060-0812, Japan

E-mail: aishizu@med.hokudai.ac.jp 


\begin{abstract}
Obesity is a state in which chronic low-grade inflammation persists in adipose tissues. Pro-inflammatory cytokines, including TNF- $\alpha$, produced by adipose tissues have been implicated as active participants in the development of obesity-related diseases. Since TNF- $\alpha$ converting enzyme (TACE) is the major factor that induces soluble TNF- $\alpha$, TACE has been noted as a pivotal regulator in this field. To reveal the role of TACE in adipose tissue inflammation, TACE-transgenic (TACE-Tg) and wild type (WT) mice were fed with high fat diet (HFD) or control diet for 16 weeks. At 13 weeks after the beginning of the diet, serum TNF- $\alpha$ and macrophage-related cytokine/chemokine levels were elevated in TACE-Tg mice fed with HFD (Tg-HFD mice), and the number of so-called crown-like adipocyte was significantly increased in adipose tissues of Tg-HFD mice at the end of the experiment. Although macrophage infiltration was not detected in the adipose tissues at this time, fibrosis was observed around the crown-like adipocytes. These findings suggested that TACE overexpression induced macrophage infiltration and subsequent fibrosis in adipose tissues under HFD regimen. The collective evidence suggested that TACE could be a therapeutic target of HFD-induced obesity-related adipose tissue inflammation.
\end{abstract}

Key words: adipose tissue inflammation, high fat diet, obesity, TACE, TNF- $\alpha$ 


\section{Abbreviations}

ADAM: a disintegrin and metalloproteinase

CD: control diet

ELISA: enzyme-linked immunosorbent assay

HE: hematoxylin and eosin

HFD: high fat diet

MCP-1: macrophage chemotactic protein-1

PKA: protein kinase A

PMA: phorbol myristate acetate

SD: standard deviation

SDS: sodium dodecyl sulfate

TACE: TNF- $\alpha$ converting enzyme

TACE-Tg: TACE transgenic

Tg: transgenic

Tg-CD: TACE-Tg mice fed with CD

Tg-HFD: TACE-Tg mice fed with HFD

WT: wild type

WT-CD: WT mice fed with CD

WT-HFD: WT mice fed with HFD 


\section{Introduction}

Obesity is a growing problem that threatens the health of a large population of humans in the world. The representative life-threatening disorders in obese patients are cardiovascular diseases. Pro-inflammatory cytokines, including TNF- $\alpha$, produced by adipose tissues have been implicated as active participants in the development of obesity-related cardiovascular diseases (Hajer et al., 2008). Currently, obesity is regarded as a state in which chronic low-grade inflammation persists in adipose tissues (Lee et al., 2013).

TNF- $\alpha$ converting enzyme (TACE), also known as a disintegrin and metalloproteinase (ADAM) 17, is the major factor that induces soluble TNF- $\alpha$ (Gooz, 2010). TACE can cleave transmembrane proteins to release their extracellular domains from the cell surface. It is initially produced as a $120 \mathrm{kDa}$ inactive protein in the cytoplasm; thereafter, the N-terminus prodomain is removed by the furin proprotein-convertase at the trans-Golgi network. Consequently, the inactive TACE is converted to a $100 \mathrm{kDa}$ active form. The active form of TACE is transported to the plasma membrane and binds to its substrates on the cell surface. The effects of TACE are dependent on its diverse array of substrates, including cytokines, growth factors, and their receptors. One of the most important substrates of TACE is the membrane-bound TNF- $\alpha$. TACE can convert TNF- $\alpha$ from the membrane-bound form to the soluble form.

In terms of the critical role of TNF- $\alpha$ in obesity and adipose tissue inflammation, TACE has been noted as a pivotal regulator in this field (Menghini et al., 2013). It was shown that TACE activity was significantly higher in mice fed with high fat diet (HFD) compared with chow controls (Fiorentino et al., 2010). In addition, TACE heterozygously knockout mice were protected against HFD-induced obesity (Serino et al., 2007). Furthermore, mice that lack tissue inhibitor of metalloproteinase 3, 
Yuki Matsui et al.

an endogenous inhibitor of ADAM families, showed an accelerated development of complications of obesity under HFD regimen (Menghini et al., 2009; Fiorentino et al., 2010). The collective findings suggested that TACE could be a possible therapeutic target of obesity-related diseases (Menghini et al., 2013). In the present study, in order to determine how excessive expression and activation of TACE could contribute to adipose tissue inflammation, TACE-transgenic (TACE-Tg) mice and wild type (WT) mice were fed with HFD or control diet (CD). 


\section{Materials and Methods}

\section{Mice}

TACE-Tg mice established in C57BL/6 line (Fukaya et al., 2013) were used. The expression of transgene, which is a heterozygously inserted mouse TACE cDNA, is driven by the $\beta$-actin promoter; therefore, the transgene-derived TACE is expressed ubiquitously. In order to distinguish from the endogenous TACE, Flag tag was connected to the 3' region of the transgene. For controls, WT C57BL/6 mice were used. Experiments using mice were permitted by the Animal Use and Care Committee (Permission No. 08-0367) and done in accordance with the Guidelines for the Care and Use of Laboratory Animals in Hokkaido University.

\section{Diet regimen}

Both TACE-Tg and WT mice (male, 12 weeks old) were fed with HFD (HFD-60, Oriental Yeast, Sapporo, Japan) or CD (AIN-93G, Oriental Yeast) for 16 weeks. The proportion of lipid-based calorie in HFD and CD was $60 \%$ and $10 \%$, respectively. Each group, namely TACE-Tg mice fed with HFD (Tg-HFD mice), TACE-Tg mice fed with CD (Tg-CD mice), WT mice fed with HFD (WT-HFD mice), and WT mice fed with CD (WT-CD mice), included more than 10 mice.

\section{Primary culture of hepatocytes}

Hepatocytes were isolated from TACE-Tg mice and cultured as previously described (Mei et al., 2011; Tomaru et al., 2012). In order to activate TACE, $0.5 \mathrm{mM}$ palmitic acid was added to the medium for $30 \mathrm{~min}$ at $37^{\circ} \mathrm{C}$.

\section{Immunoblotting}


Samples were homogenized in lysis buffer $[0.1 \%$ sodium dodecyl sulfate (SDS), $1 \quad \% \quad$ Nonidet-P40, $0.5 \quad \% \quad$ sodium deoxycholate, $100 \quad \mu \mathrm{g} / \mathrm{ml}$ phenylmethylsulphonyl fluoride, $1 \mathrm{mM}$ sodium orthovanadate, protease inhibitor cocktail (Complete Mini, Roche, Basel, Switzerland)]. The lysates, which were adjusted to range from 10 to $40 \mu \mathrm{g} /$ lane, were fractionated on $7.5 \%$ SDS polyacrylamide gel and then transferred onto PVDF membranes (GE Healthcare, Buckinghamshire, UK). After blocking by TBS-T $(0.1 \%$ Tween-20 in Tris-buffered saline) containing $2 \%$ non-fat milk, the membranes were incubated overnight with the first antibody at $4{ }^{\circ} \mathrm{C}$. After 3 times wash by TBS-T, the membranes were next incubated overnight with 1:25000 dilution of peroxidase-labelled secondary antibodies (GE Healthcare) at $4{ }^{\circ} \mathrm{C}$. Protein bands were detected using ECL Advance Western Blotting Detection kit (GE Healthcare).

\section{Antibodies}

Antibodies used in this study were anti-Flag (Sigma-Aldrich, St. Louis, MO), anti-F4/80 (Abcam, Tokyo, Japan), anti-protein kinase A (PKA) (Cell Signaling Technology, Tokyo, Japan), anti-phosphorylated PKA (Cell Signaling Technology) antibodies.

\section{Measurement of serum TNF- $\alpha$ and macrophage-related cytokines/chemokines}

At 13 weeks after the beginning of the diet, peripheral blood samples were obtained from 3 mice and then the sera were mixed in each group. The serum concentration of TNF- $\alpha$ and macrophage-related cytokines/chemokines, including IFN- $\gamma$, IL-1 $\beta$, and macrophage chemotactic protein-1 (MCP-1), was measured by enzyme-linked immunosorbent assay (ELISA) in Genetic Lab (Sapporo, Japan). 
Yuki Matsui et al.

\section{Measurement of serum leptin and adiponectin}

At the end of the experiment, peripheral blood samples were obtained from all mice. The serum concentrations of leptin and adiponectin were determined using each corresponding ELISA kit (leptin: R\&D Systems, Mineapolis, MN; adiponectin: Millipore, Billerica, MA) according to the manufacturer's protocol.

\section{Statistics}

Data were presented as mean \pm standard deviation (SD). Student's $t$-test was applied for statistical analysis. The $p$-value of less than 0.05 was considered to be significant. 


\section{Results and Discussion}

\section{Activation of transgene-derived TACE by palmitic acid}

Since the transgene-derived TACE was expressed as the $120 \mathrm{kDa}$ inactive form, no spontaneous phenotype appeared in TACE-Tg mice (Fukaya et al., 2013). We previously demonstrated that subcutaneous injection of phorbol myristate acetate (PMA) could increase TACE activity in the skin of TACE-Tg mice compared to WT mice, which was caused by the conversion of the transgene-derived inactive TACE to the active form (Fukaya et al., 2013). Since the endogenous TACE was equally expressed in TACE-Tg and WT mice and similarly converted to the active form by PMA (Fukaya et al., 2013), the conversion of the transgene-derived TACE into the active form could be considered to reflect the higher TACE activity in the PMA-treated TACE-Tg mice than in the PMA-treated WT mice.

In this study, we first determined if the transgene-derived TACE could be activated by lipid overload using primary culture cells derived from TACE-Tg mice. Palmitic acid is a major saturated fatty acid contained in various foods. When palmitic acid was added to the medium for the primary culture of TACE-Tg hepatocytes, the 100 $\mathrm{kDa}$ active form of TACE was produced (Figure 1a). This finding suggested that the prodomain of transgene-derived inactive TACE could be cleaved by excessive uptake of lipids, including palmitic acid. Therefore, Tg-HFD mice could be considered as models with abundant active TACE.

\section{High level of serum TNF- $\alpha$ in Tg-HFD mice}

TACE-Tg and WT mice were fed with HFD or CD for 16 weeks. Serum TNF- $\alpha$ level was measured at 13 weeks after the beginning of the diet. It was markedly elevated in Tg-HFD mice compared with other groups, including Tg-CD mice, WT-HFD mice, 
and WT-CD mice (Figure 1b). These findings confirmed that the transgene-derived TACE could be activated by HFD in TACE-Tg mice; thus, Tg-HFD mice were regarded as suitable models with abundant active TACE.

\section{Increased serum levels of macrophage-related cytokines/chemokines in Tg-HFD mice}

Since the feature of adipose tissue inflammation in obesity has been shown as a low-grade macrophage infiltration with circulating macrophage-related cytokines and chemokines (Surmi and Hasty, 2008), the serum concentrations of IFN- $\gamma$, IL-1 $\beta$, and MCP-1 were determined. At 13 weeks after the beginning of the diet, the serum concentrations of these molecules were higher in Tg-HFD mice than those in other groups of mice (Figure 2). These findings suggested the presence of macrophage infiltration into adipose tissues in Tg-HFD mice.

\section{Less gain in body weight and fat weight of TACE-Tg mice given HFD}

The chronological weight gain of Tg-HFD mice was lower than that of WT-HFD mice, though it was equivalent between Tg-CD mice and WT-CD mice (Figure 3a). At the end of the experiment, the increase in body weight of TACE-Tg was significantly lower than that of WT mice (Figure 3b). Autopsy revealed lower weights especially of adipose tissues around the testis and the back of TACE-Tg mice compared with WT mice (Figure 3c).

\section{Histological findings of adipose tissues of Tg-HFD mice}

In order to confirm the above findings, further histological examination was conducted. Interestingly, the number of comparatively small adipocyte, so-called crown-like adipocyte which diameter is less than $10 \mu \mathrm{m}$, was significantly greater in the adipose tissues of Tg-HFD mice than WT-HFD mice (Figures $4 \mathrm{a}$ and $4 \mathrm{~b}$ ). Since the 
relation of crown-like adipocytes with macrophage infiltration has been shown (Strissel et al., 2007), we conducted immunohistochemistry using the marker for macrophage, F4/80. However, macrophage infiltration was not detected in the adipose tissues of Tg-HFD mice at this time (Figure 4c). On the other hand, Masson trichrome staining demonstrated an increase in collagen fibers around the crown-like adipocytes (Figure 4d). We speculated the occurrence of macrophage infiltration into the adipose tissues in Tg-HFD mice at an earlier period in the experiment, and that fibrosis replaced the infiltration at the end of the experiment. This result is consistent with our previous report that demonstrated the accelerated fibrosis as one of the characteristics of TACE-Tg mice (Fukaya et al., 2013).

\section{Assessments for adipocyte-related cytokines}

In order to rule out the possibility that TACE-Tg mice detested taking HFD, the serum concentrations of adipocyte-related cytokines were determined. At the end of the experiment, the serum leptin levels were significantly increased while the adiponectin levels were significantly decreased by HFD in TACE-Tg mice as same as in WT mice (Figure 5). These findings suggested that TACE-Tg and WT mice were fed equivalently during the experiment.

\section{No promotion of adipolysis in Tg-HFD mice}

Next, we considered another possibility that adipolysis was promoted in Tg-HFD mice. It has been shown that adipolysis was mediated by cathecholamine-induced phosphorylation of PKA (Holm, 2003). When the phosphorylation of PKA was examined, it was rather suppressed in the adipose tissues of Tg-HFD mice compared with the other groups (Figure 6). These findings ruled out the promotion of adipolysis in Tg-HFD mice. 
Yuki Matsui et al.

TACE could be a possible therapeutic target of obesity-related adipose tissue inflammation

The collective evidence suggested that TACE could be a possible therapeutic target of HFD-induced obesity-related adipose tissue inflammation. Recently, treatment with a TACE inhibitor, Marimastat, has shown to reverse steatosis in mouse models of diet-induced obesity (de Meijer et al. 2011); therefore, this is consistent with our findings. In order to prevent the development of life-threatening events in obese patients, further in vivo studies are needed to demonstrate the efficacy and safety of TACE inhibitors.

\section{Author contributions}

Y.M., A.M, T.I., and S.F. performed the experiments. U.T. and A.I. designed the research. U.T., H.M., T.A., and A.I. analyzed the data, and A.I. wrote the manuscript.

\section{Additional Information}

Competing financial interests: The authors declare no competing financial interests. 


\section{References}

de Meijer, V.E, Le, H.D., Meisel, J.A., Sharma, A.K., Popov, Y., Puder, M., 2011. Tumor necrosis factor $\alpha$-converting enzyme inhibition reverses hepatic steatosis and improves insulin sensitivity markers and surgical outcome in mice. PLoS One 6, e25587.

Fiorentino, L., Vivanti, A., Cavalera, M., Marzano, V., Ronci, M., Fabrizi, M., Menini, S., Pugliese, G., Menghini, R., Khokha, R., Lauro, R., Urbani, A., Federici, M., 2010. Increased tumor necrosis factor $\alpha$-converting enzyme activity induces insulin resistance and hepatosteatosis in mice. Hepatology 51, 103-110.

Fukaya, S., Matsui, Y., Tomaru, U., Kawakami, A., Sogo, S., Bohgaki, T., Atsumi, T., Koike, T., Kasahara, M., Ishizu, A., 2013. Overexpression of TNF- $\alpha$-converting enzyme in fibroblasts augments dermal fibrosis after inflammation. Lab Invest 93, 72-80.

Gooz, M., 2010. ADAM-17: the enzyme that does it all. Crit Rev Biochem Mol Biol 45, 146-169.

Hajer, G.R., van Haeften, T.W., Visseren, F.L., 2008. Adipose tissue dysfunction in obesity, diabetes, and vascular diseases. Eur Heat J 29, 2959-2971.

Holm, C., 2003. Molecular mechanisms regulating hormone-sensitive lipase and lipolysis. Biochem Soc Trans 31, 1120-1124.

Lee, H., Lee, I.S., Choue, R., 2013. Obesity, inflammation and diet. Pediatr 
Gastroenterol Hepatol Nutr 16, 143-152.

Mei, S., Ni, H.M., Manley, S., Bockus, A., Kassel, K.M., Luyendyk, J.P., Copple, B.L., Ding, W.X., 2011. Differential roles of unsaturated and saturated fatty acids on autophagy and apoptosis in hepatocytes. J Pharmacol Exp Ther 339, 487-498.

Menghini, R., Menini, S., Amoruso, R., Fiorentino, L., Casagrande, V., Marzano, V., Tornei, F., Bertucci, P., Iacobini, C., Serino, M., Porzio, O., Hribal, M.L., Folli, F., Khokha, R., Urbani, A., Lauro, R., Pugliese, G., Federici, M., 2009. Tissue inhibitor of metalloproteinase 3 deficiency causes hepatic steatosis and adipose tissue inflammation in mice. Gastroenterology 136, 663-672.

Menghini, R., Fiorentino, L., Casagrande, V., Lauro, R., Federici, M., 2013. The role of ADAM17 in metabolic inflammation. Atherosclerosis 228, 12-17.

Serino, M., Menghini, R., Fiorentino, L., Amoruso, R., Mauriello, A., Lauro, D., Sbraccia, P., Hribal, M. L., Lauro, R., Federici, M., 2007. Mice heterozygous for tumor necrosis factor- $\alpha$ converting enzyme are protected from obesity-induced insulin resistance and diabetes. Diabetes 56, 2541-2546.

Strissel, K.J., Menghini, R., Fiorentino, L., Amoruso, R., Mauriello, A., Lauro, D., Sbraccia, P., Hribal, M.L., Lauro, R., Federici, M., 2007. Adipocyte death, adipose tissue remodeling, and obesity complications. Diabetes 56, 2910-2918.

Surmi, B.K., Hasty, A.H., 2008. Macrophage infiltration into adipose tissues: initiation, propagation and remodeling. Future Lipidol 3, 545-556. 
Yuki Matsui et al.

Tomaru, U., Takahashi, S., Ishizu, A., Miyatake, Y., Gohda, A., Suzuki, S., Ono, A., Ohara, J., Baba, T., Murata, S., Tanaka, K., Kasahara, M., 2012. Decreased proteasomal activity causes age-related phenotypes and promotes the development of metabolic abnormalities. Am J Pathol 180, 963-972. 
Yuki Matsui et al.

a

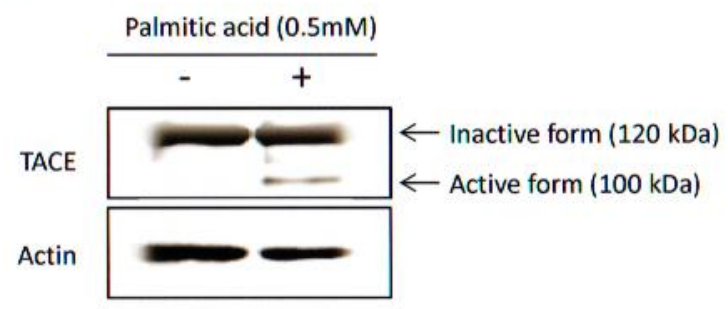

b

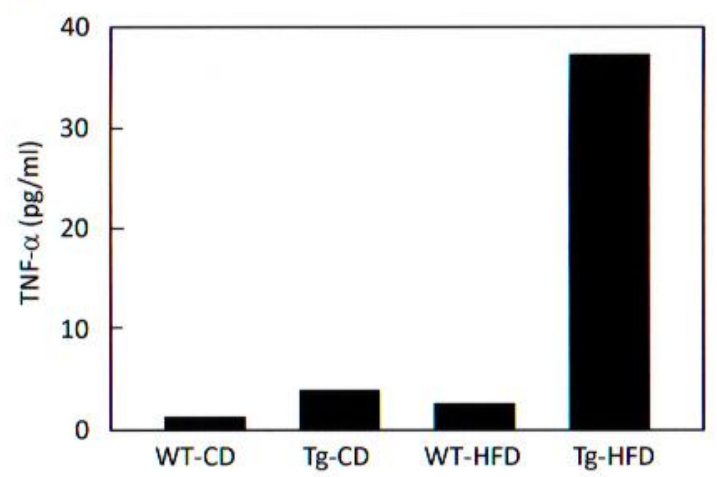

\section{Figure 1}

(a) Activation of transgene-derived TACE. TACE-Tg hepatocytes were cultured with or without $0.5 \mathrm{mM}$ palmitic acid for $30 \mathrm{~min}$ and then subjected to immunoblotting for the transgene-derived TACE, which could be detected by the anti-Flag antibody. The inactive and active forms of TACE were detected as the upper (120 kDa) and lower (100 $\mathrm{kDa}$ ) bands, respectively. Actin was employed as an internal control. Experiments were carried out 2 times independently, and similar results were reproduced. The representative results are shown. (b) Serum TNF- $\alpha$ level. Peripheral blood was obtained from 3 mice in each group at 13 weeks after the beginning of the diet. Sera were separated by centrifugation and then combined in each group. 
Yuki Matsui et al.
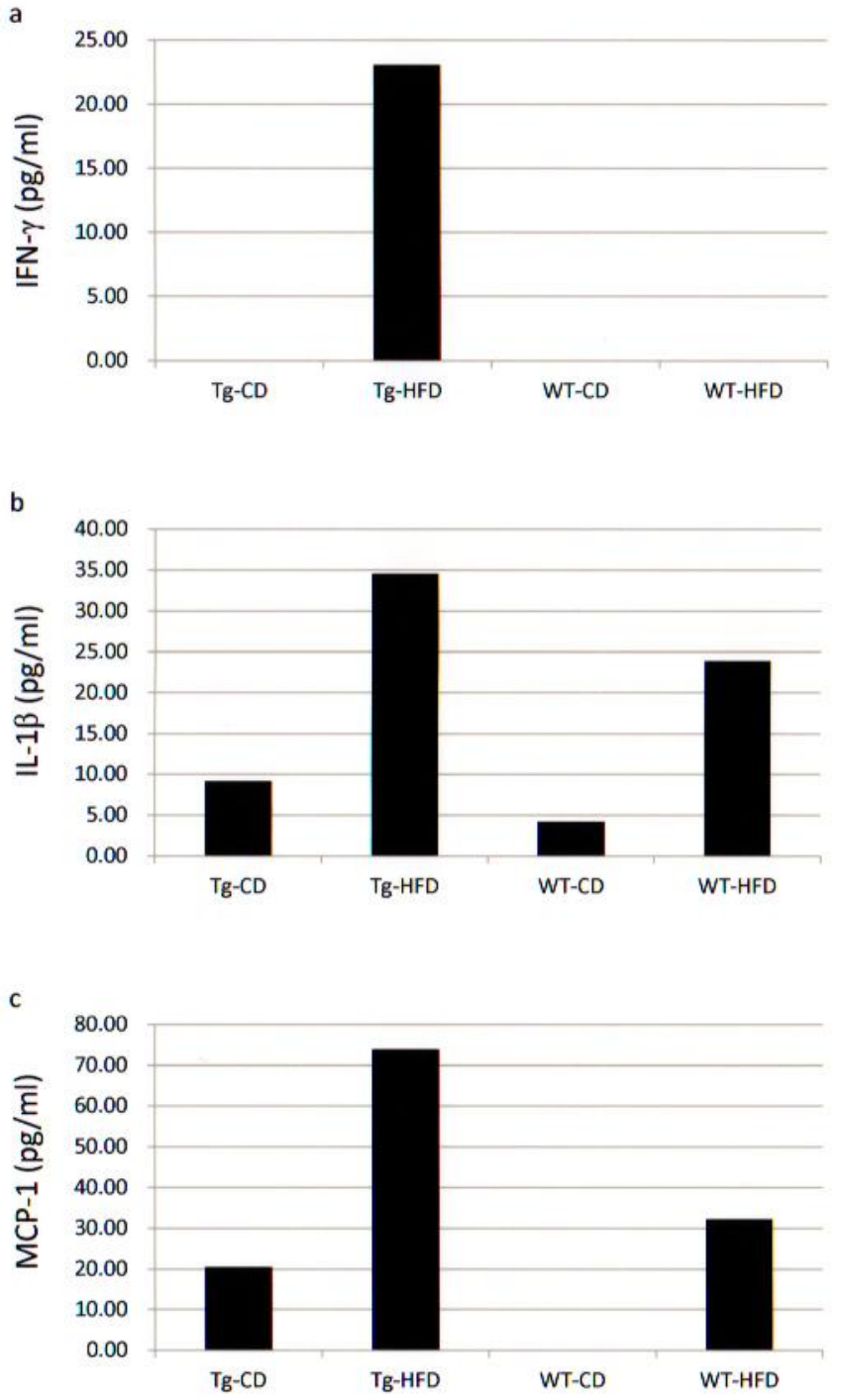

\section{Figure 2}

Serum levels of IFN- $\gamma$ (a), IL-1 $\beta$ (b), and MCP-1 (c). Peripheral blood was obtained from 3 mice in each group at 13 weeks after the beginning of the diet. Sera were separated by centrifugation and then combined in each group. 
a
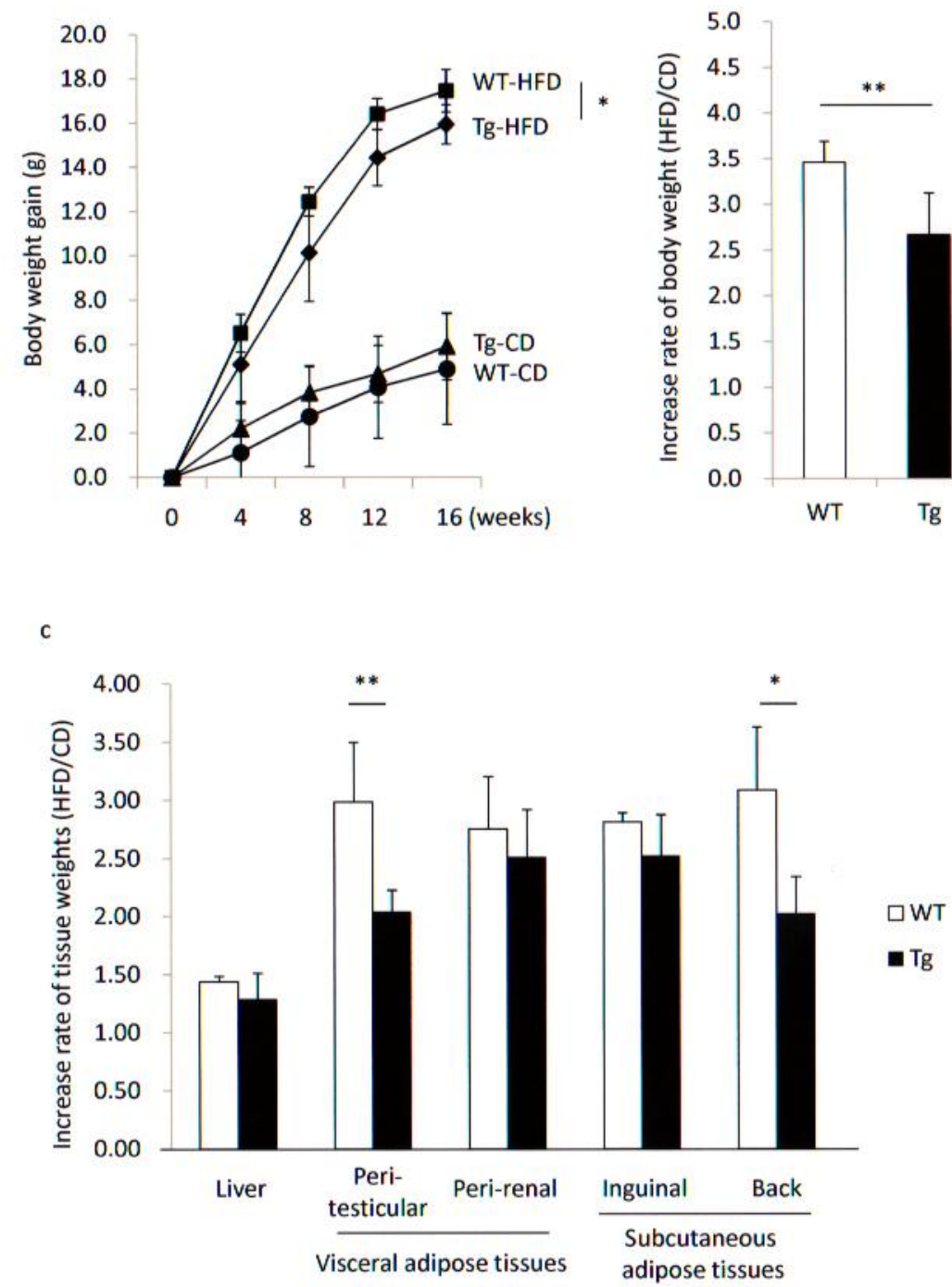

\section{Figure 3}

(a) Chronological body weight change. Body weight was measured once a week. Every 4 weeks, data were plotted. The numbers of mice used were as follows: WT-CD $\mathrm{n}=10$, WT-HFD $\mathrm{n}=14, \mathrm{Tg}-\mathrm{CD} \mathrm{n}=13$, and Tg-HFD $\mathrm{n}=13$. (b) Increase in body weight at the end of the experimental period. The weight of a mouse in the HFD group was divided by the mean weight value of $\mathrm{CD}$ group mice at the end of the experimental period. (c) Comparison of tissue weight. Weight of tissues, including the liver, visceral adipose tissues represented by peri-testicular and peri-renal adipose tissues, and subcutaneous adipose tissues represented by inguinal and back adipose tissues, was measured at the end of the experimental period. ${ }^{*} p<0.05$, $* * p<0.01$. 
a

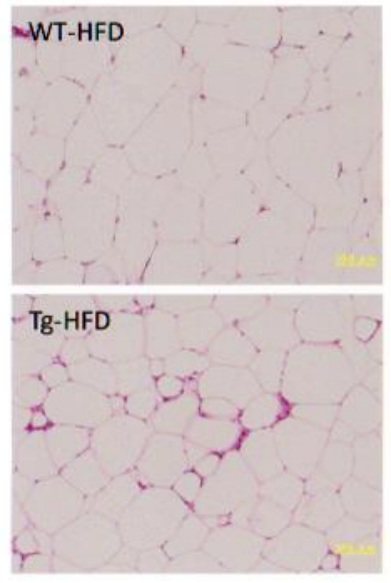

c

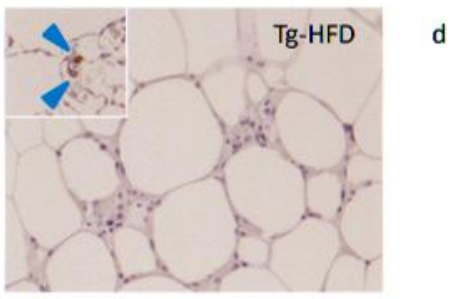

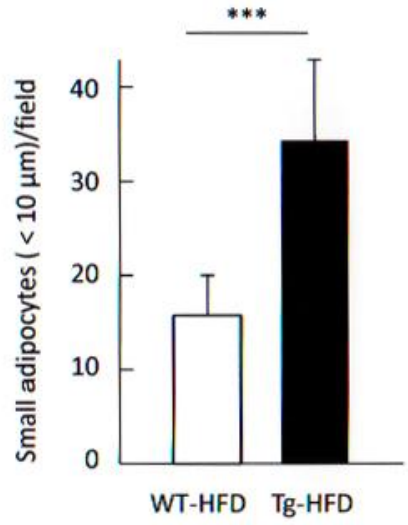

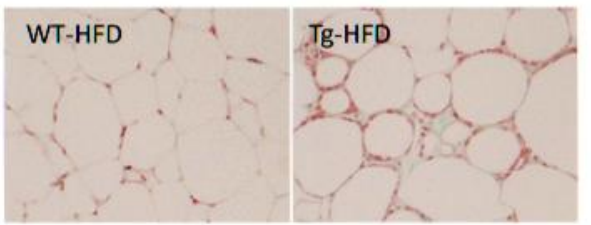

\section{Figure 4}

(a) Histology of peri-testicular adipose tissues. Upper: WT-HFD mice $(n=14)$. Lower: Tg-HFD mice $(n=13)$. The representative photos of hematoxylin and eosin (HE) staining are shown. Yellow bar: $10 \mu \mathrm{m}$. (b) Number of small adipocyte which diameter is less than $10 \mu \mathrm{m}$ per field in the peri-testicular adipose tissues. Small adipocytes were counted in 6 random fields of HE sections from WT-HFD mice $(n=3)$ and Tg-HFD mice $(\mathrm{n}=3)$. $* * * p<0.001$. (c) Immunohistochemistry using anti-F4/80 antibody. The peri-testicular adipose tissue sections of Tg-HFD mice $(n=3)$ were stained by the conventional labeled-streptoavidin biotin method. Inset: Alveolar macrophages in the lungs exhibited positive staining for F4/80 (arrow heads). Original magnification: $\times 200$. (d) Masson trichrome staining for peri-testicular adipose tissues ( $\mathrm{n}=3$, each group). Collagen fibers were stained in green. Original magnification: $\times 200$. 
Yuki Matsui et al.

a

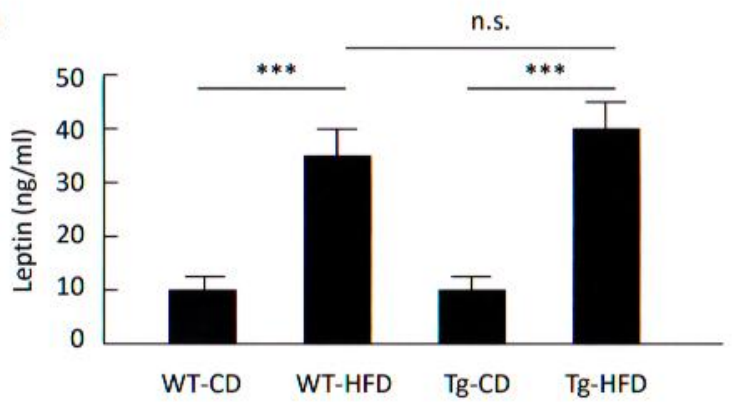

b

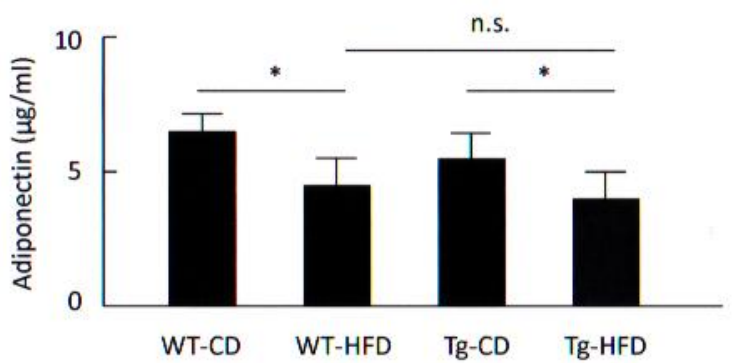

\section{Figure 5}

Serum levels of leptin (a) and adiponectin (b) were determined by each ELISA kit. Peripheral blood samples were obtained from all mice at the end of the experiment (WT-CD $\mathrm{n}=10$, WT-HFD $\mathrm{n}=14$, Tg-CD $\mathrm{n}=13$, and Tg-HFD $\mathrm{n}=13$ ). $* p<0.05$, $* * * p<0.001$, n.s.: not significant. 

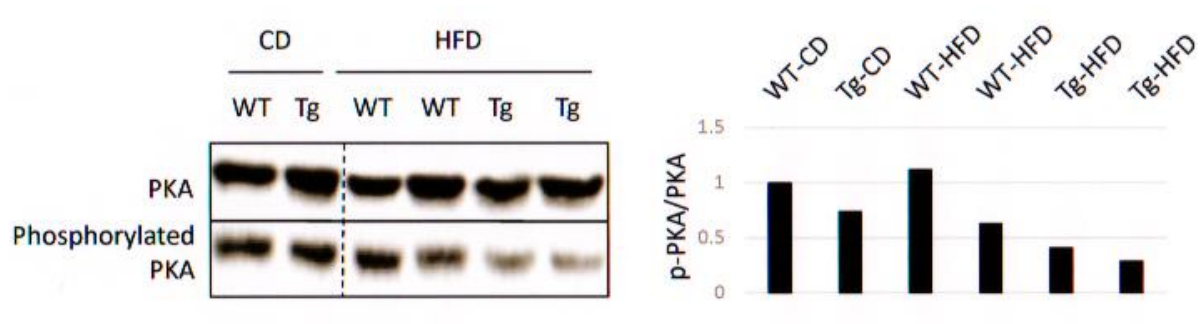

\section{Figure 6}

Immunoblotting for PKA and phosphorylated PKA. Peri-testicular adipose tissues from 1 WT-CD mouse, 1 Tg-CD mouse, 2 WT-HFD mice, and 2 Tg-HFD mice were subjected to immunoblotting. The densities of bands were quantified and then presented as the ratio of phosphorylated PKA/PKA (p-PKA/PKA). In the right panel, the value of WT-CD was set as 1. 